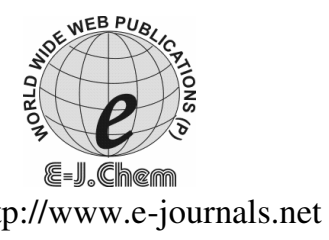

ISSN: 0973-4945; CODEN ECJHAO

E-Journal of Chemistry 2010, 7(2), 483-495

\title{
Separation of Ethanol-Water Mixture Using Type-A Zeolite Molecular Sieve
}

\author{
JUAN CAMILO DÍAZ, IVÁN DARÍO GIL-CHÁVEZ, \\ LILIANA GIRALDO ${ }^{\S}$ and JUAN CARLOS MORENO-PIRAJÁN*
}

Departamento de Ingeniería Química, Facultad de Ingeniería, Universidad de los Andes, Bogotá, Colombia.

${ }^{\S}$ Facultad de Ciencias,

Departamento de Química, Universidad Nacional de Colombia.

*Facultad de Ciencias, Departamento de Química, Grupo de Investigación en Sólidos Porosos y Calorimetría,

Universidad de Los Andes, Bogotá, Colombia.

Received 3 June 2009; Accepted 1 August 2009

\begin{abstract}
This paper presents an experimental procedure for the synthesis of a type-A zeolite molecular sieve, usable in the separation of an ethanol-water mixture. The type-A zeolite molecular sieve is obtained from a reactive mixture formed by a source of alumina, a source of silica and a promoter. Characterization of the molecular sieves obtained in this work was undertaken using X-ray diffraction (XRD), adsorption isotherms and scanning electron microscopy (SEM). The results from the characterization procedures showed that part of the product presented a pore diameter suitable for the separation of an ethanol-water mixture.
\end{abstract}

Keywords: Molecular sieve, Adsorption, Zeolite, X-ray diffraction, Scanning electron microscopy.

\section{Introduction}

The great quantity of toxic gaseous emissions produced from combustion engines may cause diseases and contribute to the global warming of the planet. This fact, coupled with the decline in global oil reserves, has made necessary the research and use of alternative renewable energy sources in order to provide a solution to these problems. Alcohol fuel is the term given to any alcohol that is mixed with petrol in order to increase the octane levels of the fuel and decrease the emission of toxic substances from combustion engines. The alcohol that is added is ethanol. The added ethanol must be of a high purity, and therefore a low water content, in order to avoid corrosion problems inside the combustion engine and also to prevent the formation of two phases which diminish the engine's efficiency ${ }^{1}$. 
The production methods of such a high purity ethanol require a large consumption of energy or the use of expensive separation agents and are reasons why more economical production methods are needed to obtain fuel of a similar efficiency. The molecular sieves can be used repeatedly and have the advantages of a high degree of dehydration, low energy consumption, permanence of the bed and the absence of harmful emissions to the environment. Zeolites are hydrated crystalline aluminosilicates that form a crystal with an ideal structure and uniform pore size following the hydration process. The pore size depends upon the type of zeolite, the cations present its structure and on the nature and conditions of the treatments that are carried out during the preparation. These treatments include calcination and leaching, among others. There are at least 39 known types of natural zeolite, whereas there are more than 100 types of synthetic zeolite. Natural zeolites are generally named after the principal mineral that they are composed of, for example mordenite. Synthetic ones are named after the initials of the researchers who developed them, preceded by the term zeolite. One example is the zeolite ZSM (Zeolite Socony-Mobil) ${ }^{2}$. Zeolites that act as molecular sieves are characterized by the separation of molecules across the adsorption area from those in the interior due to the high regularity in the size of the developed pores. Diverse types of mixtures exist which allow the synthesis of type-A zeolites. The mixtures used here were based on those reported in the literature ${ }^{3}$, where the recommended compositions of the reactant mixtures and the typical conditions of the operation process were also given. The compositions of the mixtures in terms of the constituent oxides are given in Table 1.

Table 1. Molar relations of reagents ${ }^{3}$.

\begin{tabular}{cc}
\hline Constituent oxides & Molar relations \\
\hline$\left(\mathrm{K}_{2} \mathrm{O}+\mathrm{Na}_{2} \mathrm{O}\right): \mathrm{Al}_{2} \mathrm{O}_{3}$ & $1,2-2,6$ \\
$\mathrm{SiO}_{2}: \mathrm{Al}_{2} \mathrm{O}_{3}$ & $1,8-2,5$ \\
$\mathrm{H}_{2} \mathrm{O}: \mathrm{Al}_{2} \mathrm{O}_{3}$ & $40-100$ \\
\hline
\end{tabular}

Molecular sieves are commonly used in the dehydration of gases and liquids, the separation of liquid mixtures and gaseous hydrocarbons and in various other processes. The sieves are regenerated by warming or desorption ${ }^{4}$. Generally molecular sieves composed of porous materials such as certain types of synthetic zeolites and aluminosilicates. These aluminosilicates form tunnels inside the crystalline lattice that adsorb different substances. The diameter of these tunnels (which depends on the composition of the crystalline structure) determines the size of the molecules that can pass through. This makes it possible to control the size of the molecules separated by the molecular sieve. The molecular separation also occurs due to the phenomenon of adsorption, the polarity of the involved species and the unsaturation degree. Figure 1 illustrates a schematic diagram of the separation process of an ethanol-water mixture.

\section{Experimental}

The molecular sieve was obtained from the preparation of a reactant mixture which, in suitable conditions of reaction (time and temperature of reaction), forms a crystalline structure of zeolite type-A. The mixture reactant is composed of a source of aluminum, a source of silicon and a promoter. In this project, the source of aluminum was sodium aluminate, the source of silicon was a silicate of sodium and the promoter was sodium hydroxide. From the compositions recommended ${ }^{3}$, the reactant mixtures are reported in Table 2. 


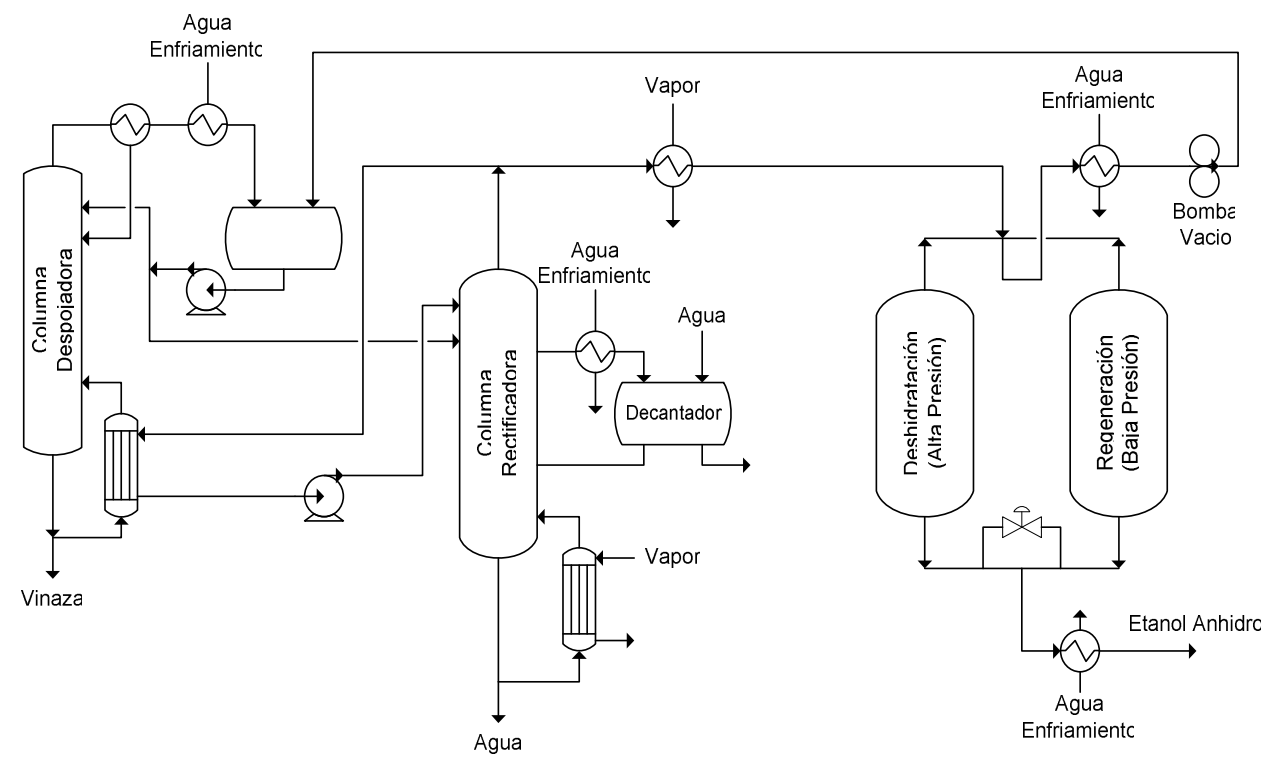

Figure 1. Generalized scheme of the dehydration process of ethanol ${ }^{5}$.

This work aims to produce a molecular sieve made of zeolitic type-A by re-creating the conditions of synthesis and subsequently studying its capacity to separate an ethanol-water mixture.

Table 2. Composition of the prepared mixtures.

\begin{tabular}{ccccccc}
\hline$\#$ & \multicolumn{7}{c}{ Molar composition mixes } & \multirow{2}{*}{ Promoter Qt. (g) } \\
\cline { 1 - 5 } Sample & $\mathrm{Na}_{2} \mathrm{O}$ & $\mathrm{Al}_{2} \mathrm{O}_{3}$ & $\mathrm{SiO}_{2}$ & $\mathrm{H}_{2} \mathrm{O}$ & $\mathrm{K}_{2} \mathrm{O}$ & \\
\hline 1 & 1,6 & 1 & 2 & 60 & 0,4 & - \\
2 & 0,58 & 1 & 2 & 60 & 1,43 & - \\
3 & 2,56 & 1 & 2 & 32,45 & - & 10 \\
4 & 2,56 & 1 & 2 & 32,45 & - & 10 \\
5 & 2,56 & 1 & 2 & 32,45 & - & 10 \\
6 & 2,38 & 1 & 1,5 & 29,49 & - & 10 \\
7 & 2,75 & 1 & 2,5 & 35,41 & - & 10 \\
8 & 2,56 & 1 & 2 & 32,45 & - & 5 \\
9 & 2,56 & 1 & 2 & 32,84 & - & 15 \\
\hline
\end{tabular}

Depending on the type of sieve to be synthesized, a certain reaction time and temperature are required. In the particular case of zeolite, suggested reaction temperatures in the literature ${ }^{3}$ range between 80 and $100{ }^{\circ} \mathrm{C}$, and reaction times between 1 and $6 \mathrm{~h}$. In this work, the temperature used in the preparation of all the samples was $100{ }^{\circ} \mathrm{C}$. In relation to samples 1 and 2, the reaction times were 3 and 2 hours respectively. The remaining samples were assigned reaction times of $5 \mathrm{~h}$. After the reaction had been carried out, two phases were generated: a white and solid one that was the zeolite and the reagents that did not react and one that was a transparent liquid that corresponded to water. These phases were separated by washing, filtering and drying. For it, a filtration is realized to the emptiness and washed with abundant water, until the filtered liquid is transparent. The resulting solid was then placed in a stove of warming and allowed to dry for a period of $12 \mathrm{~h}$, after which, the sieve was ready to be used. 


\section{$X$-ray diffraction (XRD)}

The synthesized zeolites were characterized by means of X-ray diffraction using a Rigaku Miniflex diffractometer using radiation $\mathrm{Cu} \alpha(\alpha=1.5158 \AA)$ and a secondary graphite monochromator, angle $2 \Theta$ swept and the range $2-80^{\circ}$ with a swept in the range $0.03-0,05^{\circ}$. For the XRD, the experimental spectra were compared with a theoretical diffractogram of a zeolite type-A, as reported in the literature ${ }^{6}$ and illustrated in Figure 2.

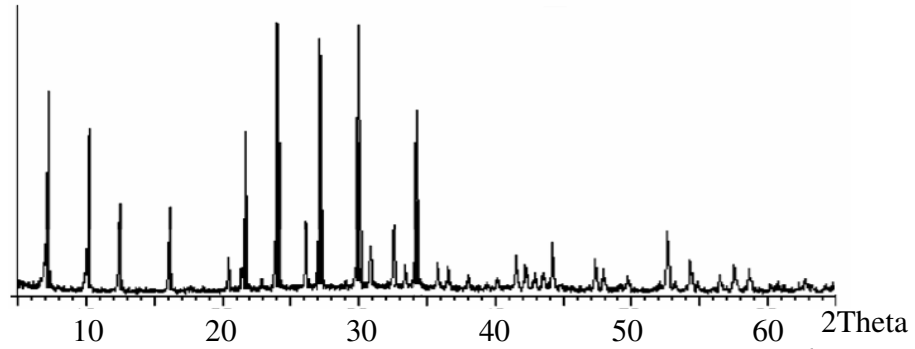

Figure 2. Theoretical diffractogram of a zeolite type- $A^{6}$.

\section{Scanning electron microscope (SEM)}

The surface morphology of the solid particles of zeolite was inspected using a Scanning Electron Microscope (SEM), FEI QUANTA 200.

\section{BET surface area and $\mathrm{N}_{2}$ adsorption isotherms to $77 \mathrm{~K}$}

Isotherms of adsorption were taken by means of a sortometer Autosorb $3 \mathrm{~b}$ Quantachrome to $77 \mathrm{~K}$. The BET area was evaluated between P/Po range of 0.05 to 0.35 .

Separation of the ethanol-water mixture using varying levels of temperature and pressure

The experimental set-up used to determine the adsorption capacity of the molecular sieves is depicted in Figure 3. As can been seen the equipment is of local construction. The procedure to determine the capacity of separation of the ethanol-water mixture involved the following steps:

a. The sieve was dried at a temperature of 300 degrees and placed in the mini-reactor.

b. Stabilization of temperature: The temperature was measured by means of a thermocouple in a feed beaker and stabilized in the range 298 to $303 \mathrm{~K}$.

c. The ethanol-water mixture was placed in the reactor (1) and the temperature and agitation levels were controlled by means of controller of which that this joined (2).

d. The mixture then entered the mini-reactor where the zeolite was formed by means of a peristaltic pump (3) and the pressure was measured (4).

e. The ethanol-water mixture entered the mini-reactor (9) where the zeolite was (8) placed between two porous membranes of glass (7). Inside was a thermostat (10) to control the experimental temperature.

f. Analytical system: Water and ethanol were analyzed by means of an on-line mass spectrometer (Omnistar ${ }^{\text {TM }}$ Pfeiffer, Vacuum QSM 200) with an enclosed ion source and a triple-mass filter, and a dual detector (Faraday and Electron Multiplier).

g. Personal computer: All data concerning the operational variables of temperature, gas signals and gas flows were continuously monitored and registered by a computer equipped with LABVIEWTM software. 


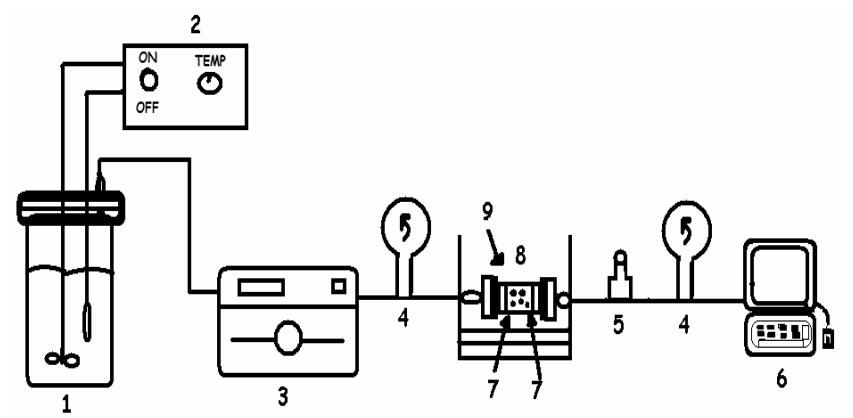

Figure 3. Experimental set-up used to determine the adsorption capacity of the molecular sieves.

\section{Results and Discussion}

Diffraction of $X$-rays

Sample preparations 1, 2 and 3, were used to establish the experimental protocol which resulted in a molecular sieve that could be applied to the separation of the ethanol-water mixture. For the other samples, some of the conditions of the process were changed in order to observe the effect of these on the resulting sieve. Qualitatively, the products obtained in samples 1 and 2 were very similar in that neither presented the formation of the two phases and the final product was a homogeneous mixture of few fluency. This did not agree with the results of the protocol undertaken in the literature ${ }^{3-16}$ where the reaction resulted in two phases that were then washed and filtered. This indicates that these procedures were not reproducible under the conditions in which the tests were undertaken. This was verified by analysis of the XRD of samples 1 and 2, which are illustrated in Figures 4 and 5 respectively.

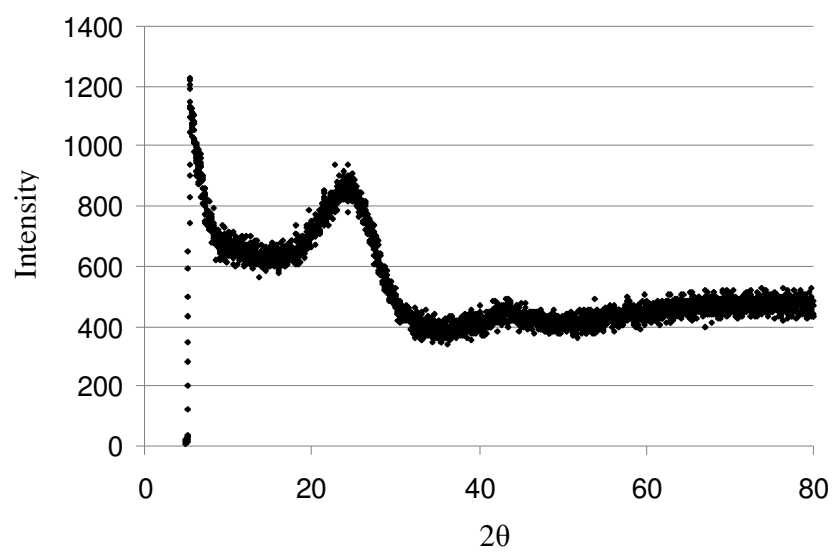

Figure 4. X-ray diffractogram of sample 1.

The absence of definite peaks indicates little crystallization, which contrasts with the results ${ }^{3,17-22}$ of in which the diffractograms and the obtained products were those of zeolite type- A. Unlike samples 1 and 2, sample 3 presented two phases at end of the reaction and after the processes of filtration, washing and drying, it was insoluble in water. X-ray diffraction of this sample observed that the crystallization changed completely and increased in comparison to the samples synthesized previously, showing major organization of the obtained product. 


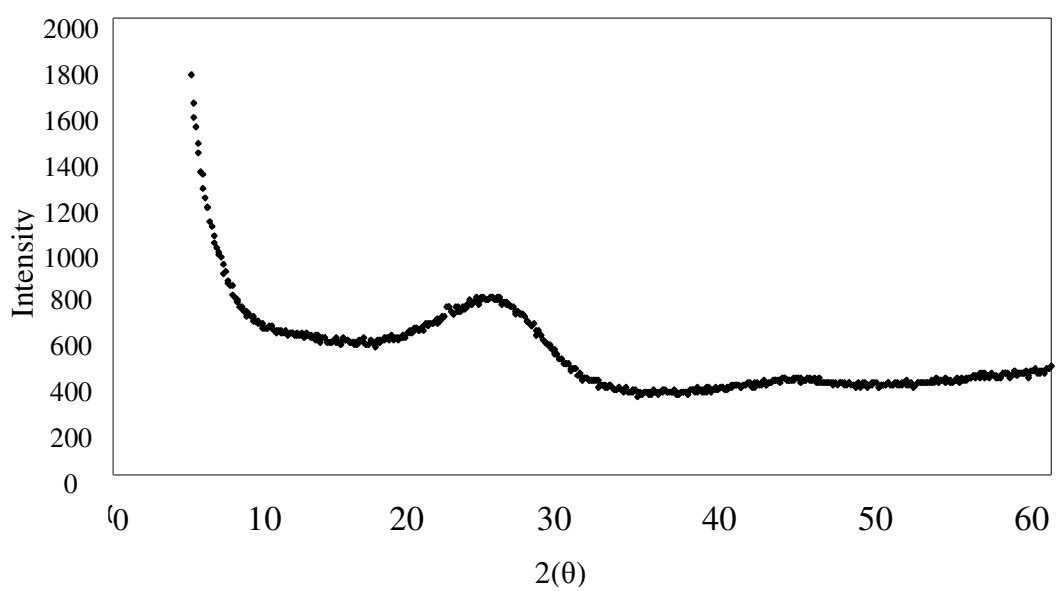

Figure 5. X-ray diffractogram for sample 2.

When the diffractogram (Figure 6), corresponding to sample 3, was compared to the diffractogram from the literature (Figure 2), it was observed that the values between $20^{\circ}$ and $35^{\circ}$ show good conformity between both graphs, fundamentally in the peak of major intensity found in the range from $25^{\circ}$ to $30^{\circ}$. This result indicates that the obtained material corresponds to zeolite type-A

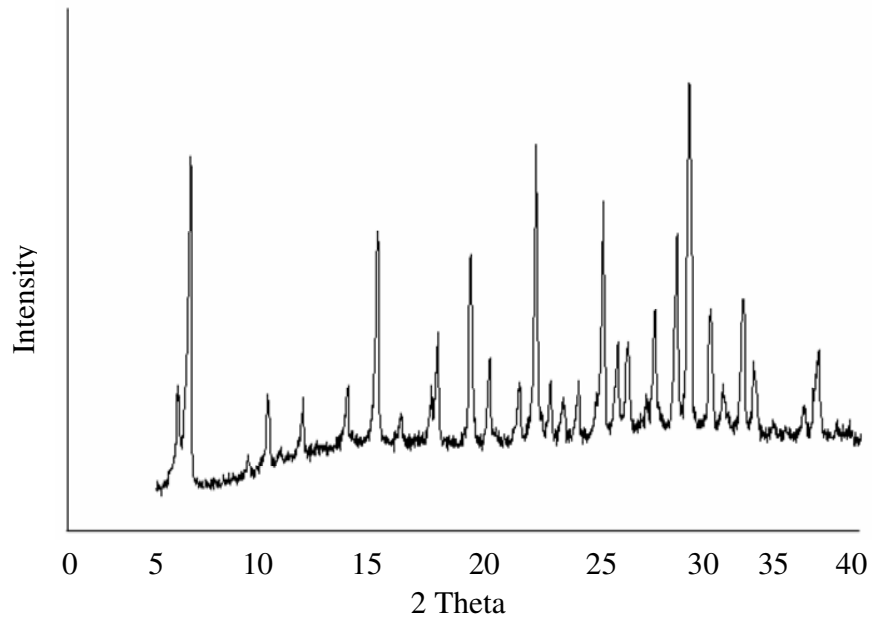

Figure 6. X-ray diffractogram of sample 3.

\section{Scanning electron microscopy}

Figure 7 illustrates the SEM of the obtained sieve from sample 3. It is possible to observe the uniformity in the composition of the surface of the sieve.

Figure 7 illustrates a large quantity of agglomerates of different sizes, (products of the metallization of the sample) and shows a great number of structures of uniform size, which are distributed along the sample. As illustrated in Figure 8, it can be appreciated that two types of structures exist on the surface of the sieve. The first one consists of a series of cubes of uniform size, and the second one of a series of small agglomerates with a spherical form of irregular surface. 


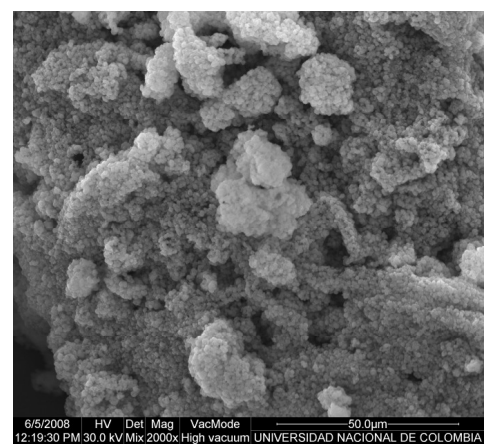

Figure 7. Scanning electron microscopy of the sieve obtained from sample 3 (Zoom 2000X).
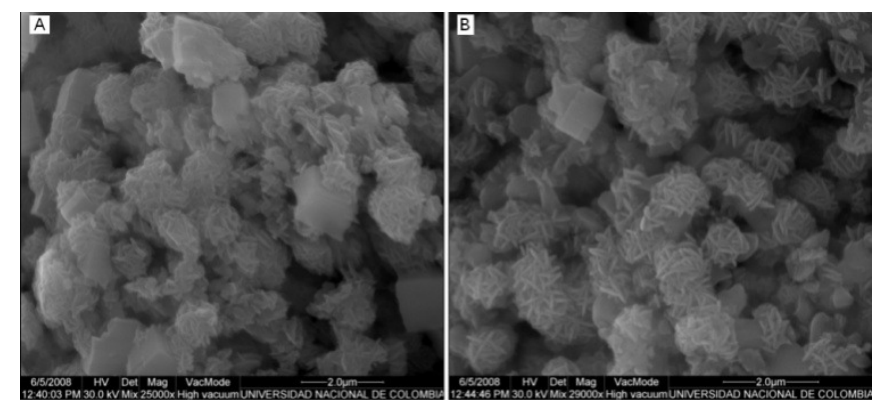

Figure 8. Scanning electron microscopy of the obtained sieve A) Zoom of 25000X, B) Zoom of 29000X.

When comparing the SEM shown in Figures 7 and 8 with that from the literature, shown in Figure 9, it can be concluded that the molecular sieve obtained was zeolite type-A.

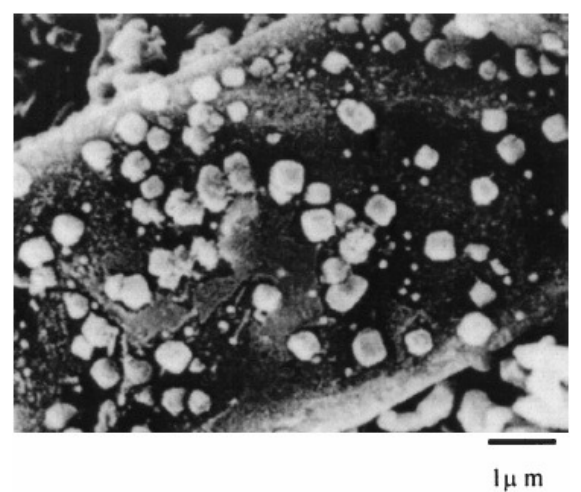

Figure 9. Theoretical scanning electron microscopy of zeolite type- $\mathrm{A}^{8}$.

For it, a filtration is realized to the emptiness and washed with abundant water, until the filtered liquid is transparent. The resulting solid was then placed in a stove of warming and allowed to dry for a period of 12 hours, after which, the sieve was ready to be used. Microstructures are amorphous structures that correspond to the reagents which did not crystallize $^{7}$. It can be implied from this that a large quantity of cubic forms in the microscopic image indicates a have present high quantity of zeolite type-A. Figure 10 illustrates a high density of cubic structures, although amorphous structures are present. 


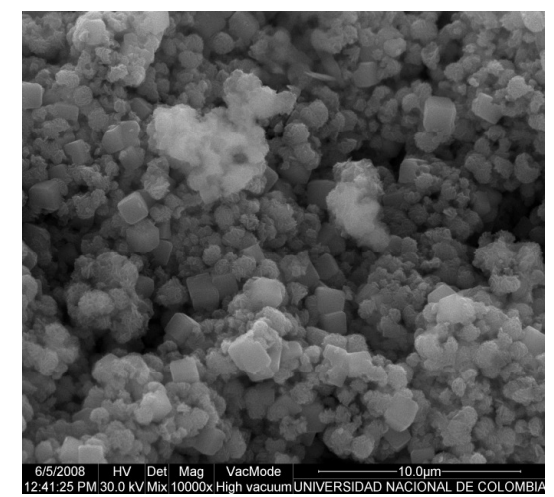

Figure 10. Scanning electron microscopy of the sieve obtained (Zoom 10000X).

\section{Adsorption of nitrogen to $77 \mathrm{~K}$}

The SEM provides a qualitative description of the surface of the sieve. A quantitative analysis was obtained across an isotherm of adsorption, as illustrated in Figure 11.

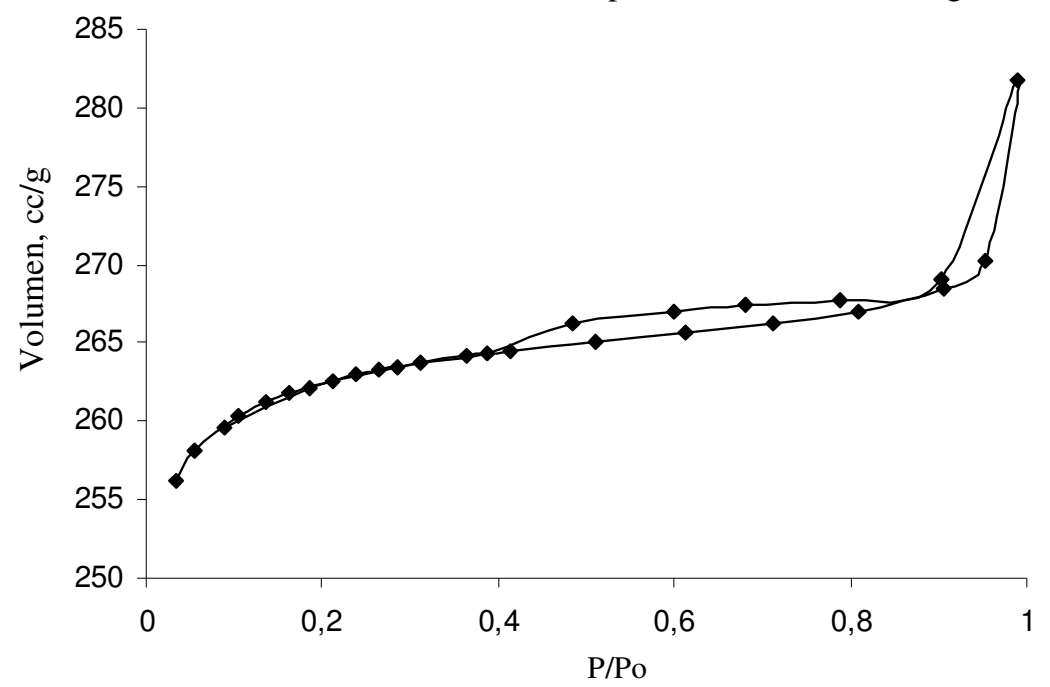

Figure 11. Adsorption isotherm of nitrogen for the sieve obtained from sample 3.

The results of the isotherm of adsorption show a superficial area evaluated by the BET model (this is the total area) of $365 \mathrm{~m}^{2} / \mathrm{g}$, whereas the representative area of micropores $\left(\mathrm{S}_{\text {micro }}\right)$ is calculated by the method $t$-plot and is of $201 \mathrm{~m}^{2} / \mathrm{g}$, the $\mathrm{V}_{\text {total }}\left(\mathrm{cm}^{3} / \mathrm{g}\right)$ of 0.56 and the Vmicro $\left(\mathrm{cm}^{3} / \mathrm{g}\right)$ of 0.21 . The isotherm shows microporosity at low relative pressures and a loop hysteresis that allows establishing the presence of mesopores. The isotherm represents uniform filling of pores at low pressures, the previous thing means that is interpreted as a uniform distribution of pores that is also characteristic of the molecular sieve. One of the principal characteristics that are desirable in a molecular sieve is that of pore diameter uniformity. The pore diameter distribution for the sieve obtained from sample 3 is shown in Figure 12. The distribution of pores illustrates that the synthesized sample mainly consisted of pores with a diameter of $4 \AA$, which is typical of zeolite type- $A$. 


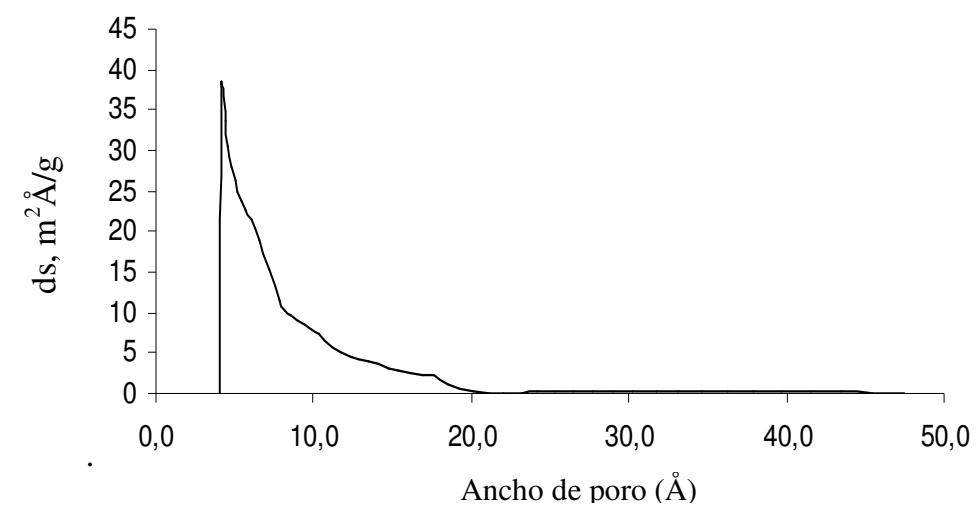

Figure 12. Pore size distribution of the sieve synthesized from sample 3.

\section{Characterization of the molecular sieve}

\section{Effect of pressure and selectivity in the separation of the ethanol-water mixture}

Figure 13 shows the effect of the feed (retentive) pressure in the range of 1 to 8 bar on the total flow (Nt) and selectivity towards dehydration of the ethanol-water $(\mathrm{S}, \mathrm{W} / \mathrm{E})$ mixture (90:10 wt \%) at $303 \mathrm{~K}$ by zeolite type-A synthesized in this study. As can be seen, while the total flow tends to rise slightly with the feed pressure, the ethanol-water selectivity shows quite a dramatic reduction.

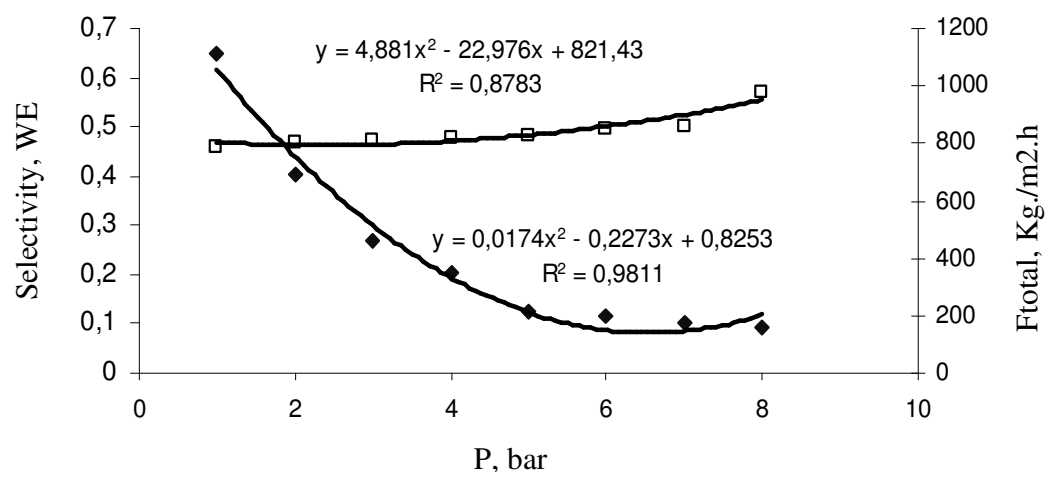

Figure 13. Evolution of total flow and selectivity with increasing feed pressure of the ethanol-water mixture.

Although the zeolite shows a high quality, water's dehydration being present, this may be confirmed by the presence of a reduced number of a large pores in non-zeolitic the zeolite layer, which might correspond with fissures, cracks or pinholes. In this way, a higher dependence of both properties on the feed pressure might reveal the presence of a larger number of large non-zeolite type-A layers. It should be emphasized that non-zeolite pores, especially the largest ones, might contribute to the PV perfomance of a membrane even at very low feed pressures, because the permeate vapor is kept at $<2$ mbar in most of the experiments and a net pressure driving force (at least 1 bar) exists across the membrane. However, to reduce their contribution, most of the experiments were carried out at a low feed pressure (1.4-1,6 bar), but higher than atmospheric pressure to prevent the liquid feed from vaporization at the highest temperatures (i.e. 363K). 


\section{Effect of feed composition}

Figure 14 illustrates the effect of the feed composition for the temperature range 298 to $303 \mathrm{~K}$ on the total flow and ethanol-water mixture for the synthesized zeolite type-A. As can be seen, the total flow is observed to increase with the water feed composition for all the surveyed temperatures. At low water composition $(20 \mathrm{~mol} \%$ or $<10 \mathrm{wt} \%)$, the total flow shows a linear trend with the water composition, while for values above $30-40 \mathrm{~mol} \%$ (20-30 wt \%), this flow tends to be a constant asymptotic value.

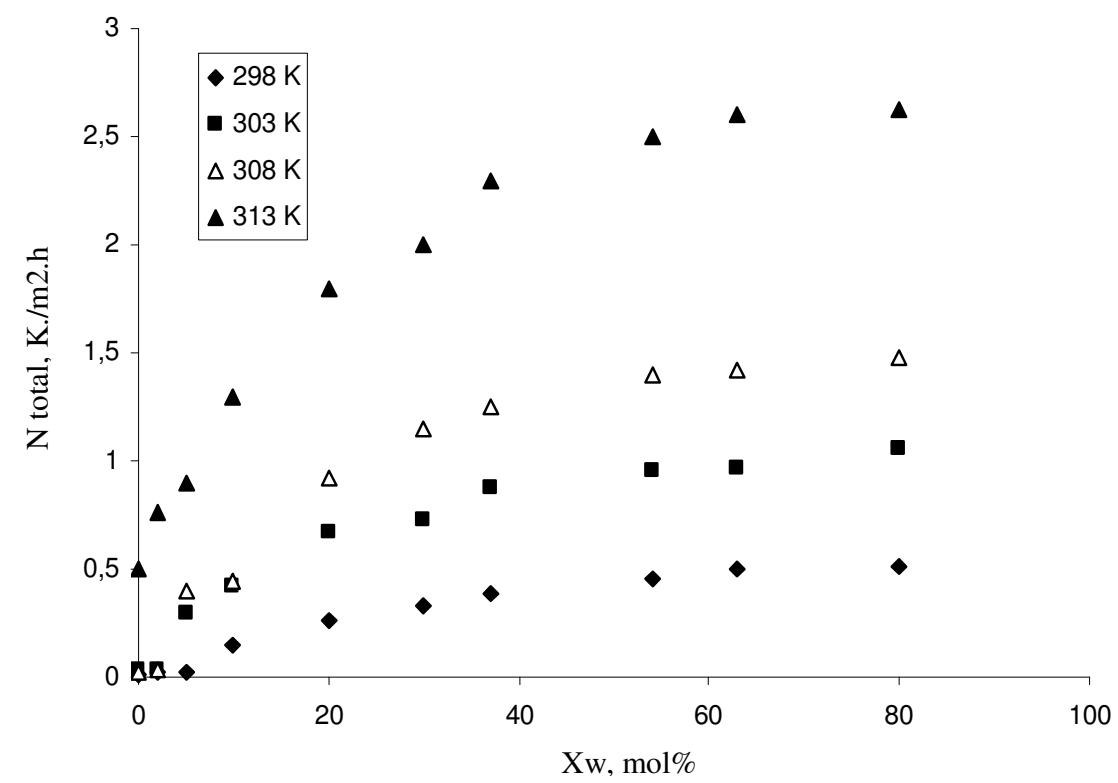

Figure 14. Evolution of total flow versus water feed with increasing temperature.

These observations illustrate the great affinity for water that the zeolite synthesized here has, which is due to its high hydrophilic character. Thus the active zeolite layer may preferentially adsorb water over ethanol and as a result, water flow through the membrane may remain high and constant in high water compositions. On the other hand, the decrease in total flow observed for water composition $<20 \mathrm{~mol} \%$ may be related to the decrease in the water feed activity. In fact, the trend depicted in Figure 14 is qualitatively similar to the form of a singlesite Langmuir isotherm, which relates surface coverage with partial pressure at equilibrium. This analogy might imply a relevant contribution of water adsorption equilibrium at the liquid feed/zeolite surface on PV performance of the separation system. On the other hand, Figure 15 shows the effect of the feed composition in the selectivity of zeolite type-A for the temperature range being studied. As can be seen, the selectivity shows a maximum with the water feed composition in the range 10 to $20 \mathrm{~mol} \%$ (5-10 wt\%) which is in agreement with that reported by the literature, with selectivity values up to $1400 \pm 70$ at $303 \mathrm{~K}$ in the present study. The presence of a maximum in the plot of ethanol-water selectivity with the water composition might be explained by the definition of selectivity, $\alpha_{\mathrm{w} / \mathrm{E}}=\left[\mathrm{y}_{\mathrm{w}} /\left(1-\mathrm{y}_{\mathrm{w}}\right)\right] /\left[\left[\left(1-\mathrm{x}_{\mathrm{w}}\right) / \mathrm{x}_{\mathrm{w}}\right]\right.$.

For water compositions $<20 \mathrm{~mol} \%(10 \mathrm{wt} \%)$, water and ethanol flows show a dramatic increase, which implies an increase in the selectivity. Above $20 \mathrm{~mol} \%$ (10 wt $\%)$, water and ethanol flows tend to show a steady value, which involves the reduction of selectivity with water composition according to the function $\mathrm{f}\left(\mathrm{x}_{\mathrm{w}}\right)=\left[\mathrm{y}_{\mathrm{w}} /\left(1-\mathrm{y}_{\mathrm{w}}\right)\right] /\left[\left[\left(1-\mathrm{x}_{\mathrm{w}}\right) / \mathrm{x}_{\mathrm{w}}\right]\right.$. 


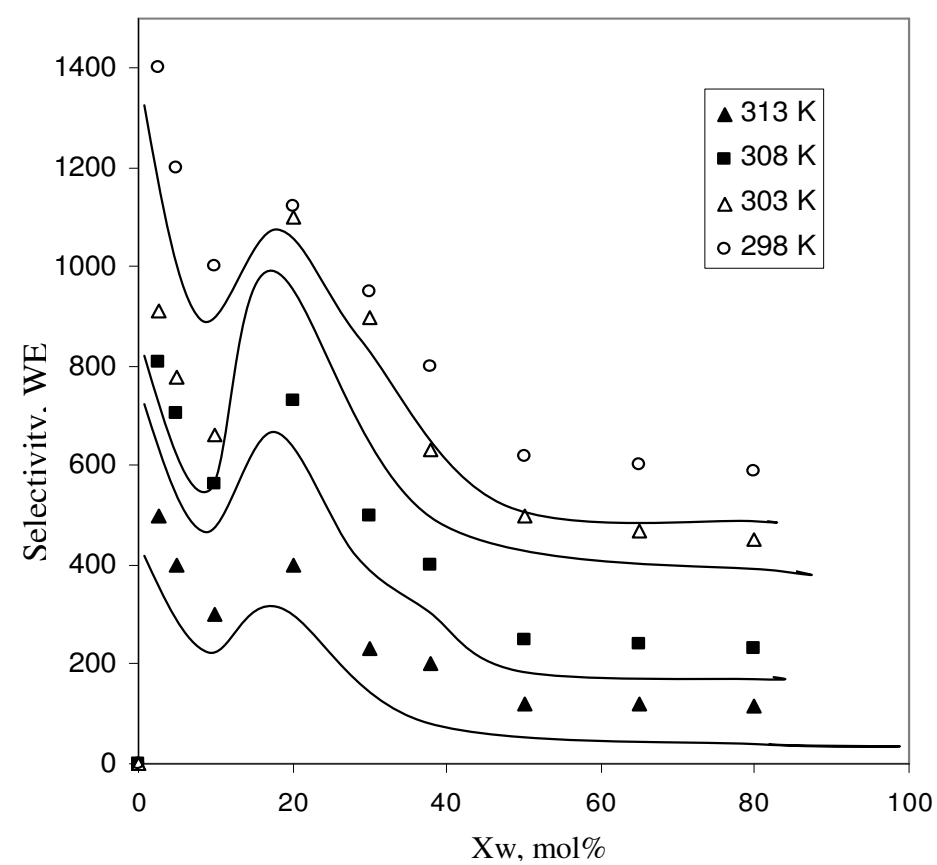

Figure 15. Evolution of ethanol-water selectivity with water feed composition (molar) using zeolite type-A.

\section{Verification of the experimental protocol}

Once it was verified that the molecular sieve was functional in the separation of the ethanolwater mixture, the procedure undertaken with samples 4 and 5 obtained the same results and reproducibility. Sample 4 contained $85: 15$ wt $\%$ ethanol-water and sample 5 contained 80:20 wt \% ethanol-water. The diffractograms of samples 3, 4 and 5 are shown in Figure 16.

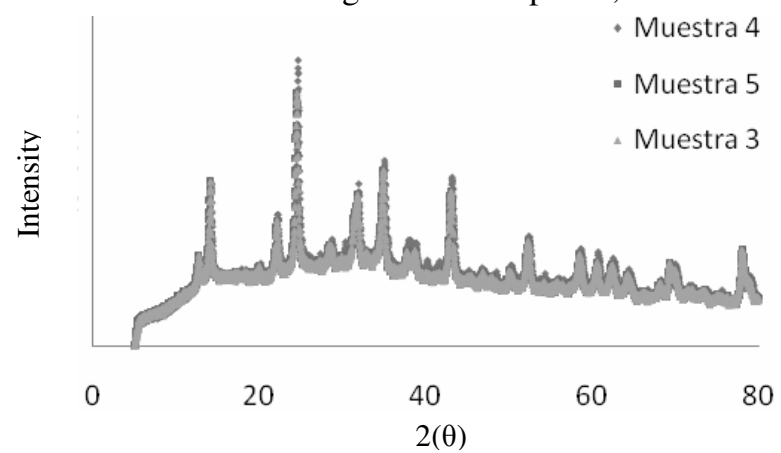

Figures 16. Comparison of the diffractograms of samples 3, 4 and 5.

This graph illustrates that the diffractograms of the samples are identical, which implies that the experimental procedure used in the production of the sieve is reproducible when the same process conditions and relative molar concentrations are used as in the preparation of sample 3. The stages of the experimental protocol used to prepare the molecular sieve which possesses the aforementioned characteristics are as follows: A reactant mixture must be prepared according to the following compositions in terms of relative molar concentrations: 
- $\mathrm{SiO}_{2}: \mathrm{Al}_{2} \mathrm{O}_{3} \rightarrow 2$

- $\mathrm{NaOH}: \mathrm{Al}_{2} \mathrm{O}_{3} \rightarrow 0.25$

- $\mathrm{H}_{2} \mathrm{O}: \mathrm{Al}_{2} \mathrm{O}_{3} \rightarrow 32.45$

1. The reagents must be added in the following way and under constant agitation. First add the sodium hydroxide to the sodium aluminate, then add the sodium silicate one drop at a time. This must be done in order to avoid the rapid agglomeration that occurs between silicon oxide and the aluminum oxide.

2. The reactant mixture must be kept at a temperature of $373 \mathrm{~K}$ and an atmospheric pressure of 0.73 atmospheres, for a period of $5 \mathrm{~h}$. This must be done in order to allow sufficient time for the crystallization of the aluminosilicates to happen in the shape of zeolite type-A.

3. It filters to the emptiness and to wash with abundant water, until the filtered liquid is transparent. This ensures that those reagents which have not reacted are removed.

4. Dry to a temperature of $343 \mathrm{~K}$ for $12 \mathrm{~h}$ in order to remove the moisture retained by the sieve from the washing process. It is advisable to work at this temperature provided that of working to major temperatures, the quantity of amorphous material that exists in the sieve can start crystallizing of not controlled form, and to change this way the properties of the sieve.

5. Store in a dry environment to prevent the sieve from becoming damp through absorption of moisture from the air.

\section{Conclusions}

The results obtained from the isotherms, SEM and XRD demonstrated that the molecular sieve synthesized was zeolite type-A. The synthesized sieve is captable of water separation of the ethanol-water mixture where it was observed that, with regards to the pressure, the selectivity diminishes while the flow of the mixture increases slightly. The total flow increases as water separation of the mixture occurs.

\section{References}

1. Ministerio de Minas y Energía 2006; Beneficios del Alcohol Carburante. http://www.minminas.gov.co/minminas/prensa.nsf/. Citado Feb. 2008

2. C N Satterfield, Heterogeneous Catalysis in Industrial Practice, R E Krieger Publishing Company, 1999.

3. Vaughan D E, Process for Direct Synthesis of Sodium - and Potassium - containing Zeolite A, United States Patent, August 13 de 1985. http://patft.uspto.gov/

4. Treybal R E, Mass Transfer Operations, $3^{\text {rd }}$ Ed. Mc Graw Hill, 1980.

5. Uyazán A M, Gil I D, Aguilar A J L, Rodríguez G and Caicedo L A, Revista Ingeniería e Investigación, 2004, 56, 49-56.

6. Chudasama C D, Sebastian J and Jasra R V, Indian Eng Chem Res., 1997, 44, 1780-1786.

8. Han Y, Hao M, Shilin Q and Feng-Shou X, Micropor Mesopor Mater., 1996, 30, 321-326.

9. Abeles B, Chen L F, Johnson J W, Israel J Chem., 1991, 19, 99-106.

10. Alfaro S, Arruebo M, Coronas J, Menendez M and Santamaría J, Micropor Mesopor Mater., 2001, 50, 195-200.

11. Baltus R E, J Membr Sci., 1997, 123, 165-184.

12. Bakker W J W, Kapteijn F and Moulin J A, ALChe J., 1997, 43(9), 2203-2214.

13. Barrer R M, Hydrothermal Chemistry of Zeolites, Academic Press, London-New York, UK-USA, 1982.

14. Coronas Ju, Falconer J L and Noble R D, ALChE J., 1981, 27, 5-12.

15. Gallego-Lizon T, Edwards E, Lobiundo G and Freitas dos Santos L, J Membr Sci., 2002, 197, 309-319. 
16. Huang C P and Rhoads E A, J Colloid Interf Sci., 1989, 131, 289-306.

17. Jafar J J and Budd P M, Micropor Mater., 1997, 12, 305-311.

18. Hans O.E. Karlsson and Gun Trägårdh J Membr Sci., 1993, 76, 121-146.

19. Lee C C, Gorte R J and Farneth W E, J Phys Chem B., 1997, 101, 3811-3817.

20. Li S, Tuan V A, Noble R D and Falconer J L, Indian Eng Chem Res., 2001, 40, 1952-1959.

21. Li S, Tuan V A, Noble R D and Falconer J L, Indian Eng Chem Res., 2001, 40, 6165-6171.

22. Myers A L and Praunitz J M, AlChE J., 2002, 48, 145-160.

23. Myers A L and Praunitz J M, AlChE J., 1965, 11, 121-130.

24. Neimark A, Physica A., 1992, 191, 258-262.

25. Rege S U and Yang R T, Chem Eng Sci., 2001, 56, 3781-3796.

26. Shah D, Kissick K, Ghorpade A, Hannah R and Bhattacharyya D, J Membr Sci., 2000, 179, 185-205. 


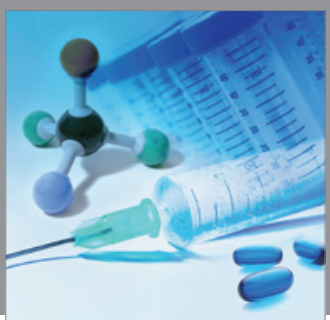

International Journal of

Medicinal Chemistry

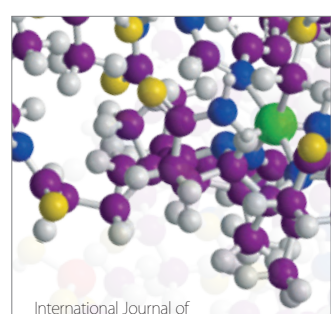

Carbohydrate Chemistry

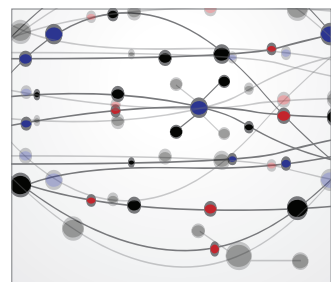

The Scientific World Journal
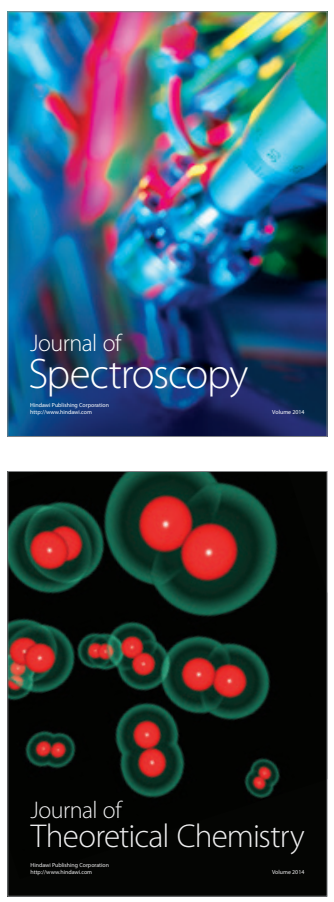
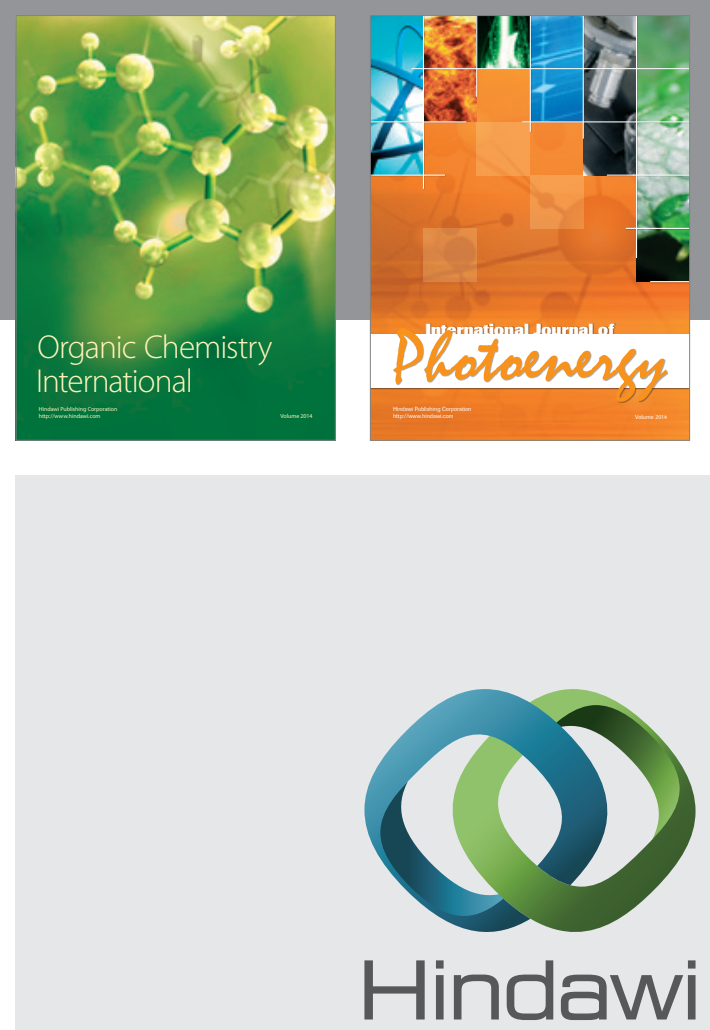

Submit your manuscripts at

http://www.hindawi.com
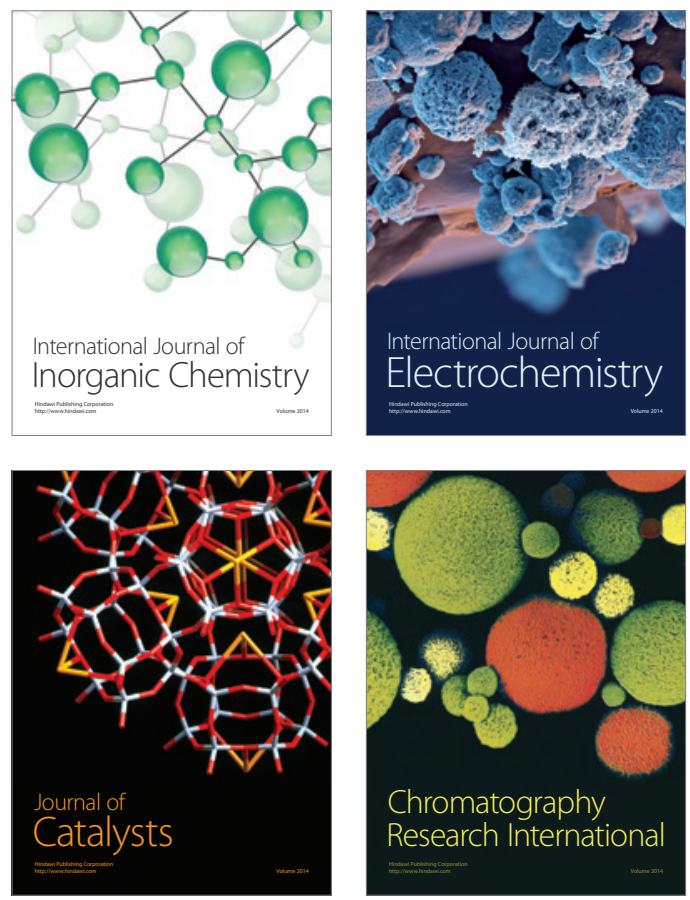
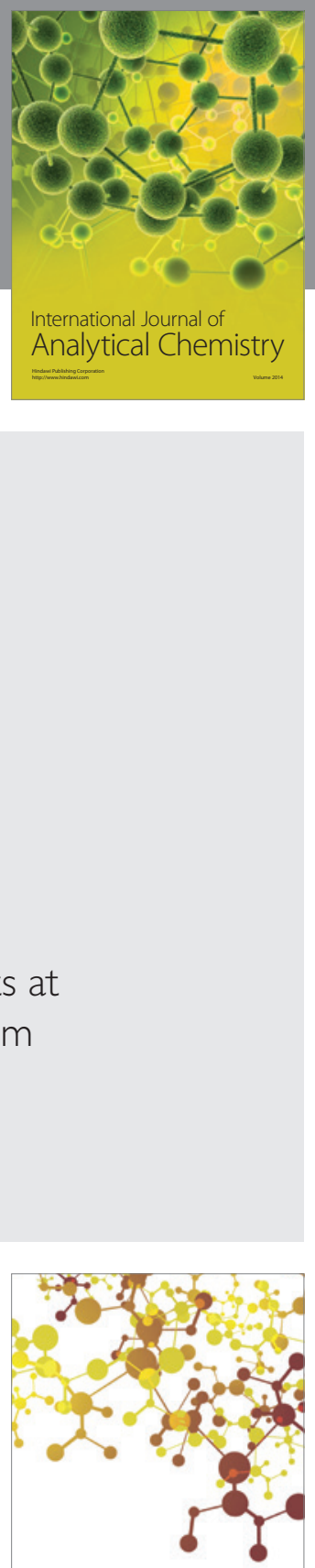

Journal of

Applied Chemistry
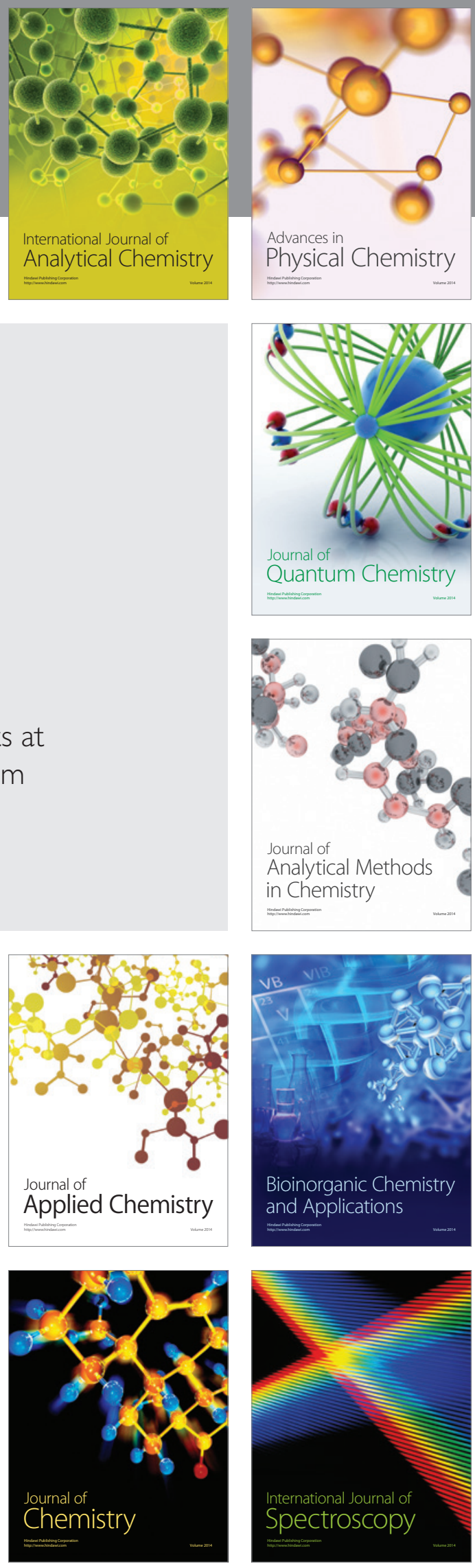